\title{
Existence and Convergence Theorems by an Iterative Shrinking Projection Method of a Strict Pseudocontraction in Hilbert Spaces
}

\author{
Kasamsuk Ungchittrakool ${ }^{1,2}$ \\ ${ }^{1}$ Department of Mathematics, Faculty of Science, Naresuan University, Phitsanulok 65000, Thailand \\ ${ }^{2}$ Centre of Excellence in Mathematics, CHE, Si Ayutthaya Road, Bangkok 10400, Thailand
}

Correspondence should be addressed to Kasamsuk Ungchittrakool; kasamsuku@nu.ac.th

Received 23 May 2013; Revised 5 October 2013; Accepted 5 October 2013

Academic Editor: Naseer Shahzad

Copyright (C) 2013 Kasamsuk Ungchittrakool. This is an open access article distributed under the Creative Commons Attribution License, which permits unrestricted use, distribution, and reproduction in any medium, provided the original work is properly cited.

\begin{abstract}
The aim of this paper is to provide some existence theorems of a strict pseudocontraction by the way of a hybrid shrinking projection method, involving some necessary and sufficient conditions. The method allows us to obtain a strong convergence iteration for finding some fixed points of a strict pseudocontraction in the framework of real Hilbert spaces. In addition, we also provide certain applications of the main theorems to confirm the existence of the zeros of an inverse strongly monotone operator along with its convergent results.
\end{abstract}

\section{Introduction}

There are several attempts to establish an iteration method to find a fixed point of some well-known nonlinear mappings, for instant, nonexpansive mapping. We note that Mann's iterations [1] have only weak convergence even in a Hilbert space (see, e.g., [2]). Nakajo and Takahashi [3] modified the Mann iteration method so that strong convergence is guaranteed, later well known as a hybrid projection method. Since then, the hybrid method has received rapid developments. For more details, the readers are referred to papers [4-23] and the references cited therein. In 2008, Takahashi et al. [18] introduced an alternative projection method, subsequently well known as the shrinking projection method, and they showed several strong convergence theorems for a family of nonexpansive mappings; see also [24]. In 2009, Aoyama et al. [25] applied the hybrid shrinking projection method along with creating some necessary and sufficient conditions to confirm the existence of a fixed point of firmly nonexpansive mapping.

Let $H$ be a real Hilbert space; a mapping $T$ with domain $D(T)$ and range $R(T)$ in $H$ is called firmly nonexpansive if

$$
\|T x-T y\|^{2} \leq\langle T x-T y, x-y\rangle, \quad \forall x, y \in D(T),
$$

nonexpansive if

$$
\|T x-T y\| \leq\|x-y\|, \quad \forall x, y \in D(T) .
$$

Throughout this paper, $I$ stands for an identity mapping. The mapping $T$ is said to be a strict pseudocontraction if there exists a constant $0 \leq k<1$ such that

$$
\begin{array}{r}
\|T x-T y\|^{2} \leq\|x-y\|^{2}+k\|(I-T) x-(I-T) y\|^{2}, \\
\forall x, y \in D(T) .
\end{array}
$$

In this case, $T$ may be called as a $k$-strict pseudocontraction. It is not hard to verify that (3) is equivalent to

$$
\begin{array}{r}
\langle x-y,(I-T) x-(I-T) y\rangle \\
\geq \frac{1-k}{2}\|(I-T) x-(I-T) y\|^{2}, \\
\forall x, y \in D(T) .
\end{array}
$$

If we set $A:=(I-T)$ that satisfies (4), then $A$ is said to be inverse strongly monotone. For such a case, $A$ may be called as $(1-k) / 2$-inverse strongly monotone (let us see Section 4). 
We use $F(T)$ to denote the set of fixed points of $T$ (i.e., $F(T)=\{x \in D(T): T x=x\}) . T$ is said to be a quasi-strict pseudocontraction if the set of fixed points $F(T)$ is nonempty and there exists a constant $0 \leq k<1$ such that

$$
\begin{array}{r}
\|T x-p\|^{2} \leq\|x-p\|^{2}+k\|x-T x\|^{2}, \\
\forall x \in D(T), \quad p \in F(T) .
\end{array}
$$

The class of strict pseudocontractions extends the class of nonexpansive mappings and firmly nonexpansive mappings. That is $T$ is nonexpansive if and only if $T$ is a 0 -strict pseudocontraction.

By definition, it is clear that

$$
\begin{aligned}
\text { firmly nonexpansive } & \Longrightarrow \text { nonexpansive } \\
& \Longrightarrow \text { strict pseudocontraction }
\end{aligned}
$$

However, the following examples show that the converse is not true.

Example 1. Let $H$ be a real Hilbert space and $\alpha \in(1, \infty)$. Define $T_{\alpha}: H \rightarrow H$ by

$$
T_{\alpha} x=-\alpha x, \quad \forall x \in H .
$$

Then, $T_{\alpha}$ is a strict pseudocontraction but not a nonexpansive mapping.

Indeed, it is clear that $T_{\alpha}$ is not nonexpansive. On the other hand, let us consider

$$
\begin{aligned}
& \left\|T_{\alpha} x-T_{\alpha} y\right\|^{2} \\
& =\|(-\alpha x)-(-\alpha y)\|^{2}=\alpha^{2}\|x-y\|^{2} \\
& =\left(1+\frac{\alpha^{2}-1}{(1+\alpha)^{2}}(1+\alpha)^{2}\right)\|x-y\|^{2} \\
& =\|x-y\|^{2}+\frac{\alpha^{2}-1}{(1+\alpha)^{2}}\|(1-(-\alpha)) x-(1-(-\alpha)) y\|^{2} \\
& =\|x-y\|^{2}+\frac{\alpha-1}{\alpha+1}\left\|\left(I-T_{\alpha}\right) x-\left(I-T_{\alpha}\right) y\right\|^{2} \\
& \leq\|x-y\|^{2}+\kappa\left\|\left(I-T_{\alpha}\right) x-\left(I-T_{\alpha}\right) y\right\|^{2}
\end{aligned}
$$

for all $\kappa \in[(\alpha-1) /(\alpha+1), 1)$. Thus, $T_{\alpha}$ is a strict pseudocontraction.

Example 2. Take $H \neq\{0\}$, and let $T=-I$ : it is not hard to verify that $T$ is nonexpansive but not firmly nonexpansive.

From a practical point of view, strict pseudocontractions have more powerful applications than nonexpansive mappings do in solving inverse problems (see Scherzer [26]). Therefore, it is important to develop theory of iterative methods for strict pseudocontractions. Within the past several decades, many authors have been devoted to the studies on the existence and convergence of fixed points for strict pseudocontractions. In 1967, Browder and Petryshyn [27] introduced a convex combination method to study strict pseudocontractions in Hilbert spaces. On the other hand, Marino and $\mathrm{Xu}$ [11] and Zhou [28] developed some iterative scheme for finding a fixed point of a strict pseudocontraction mapping.

In 2009, Yao et al. [29] introduced the hybrid iterative algorithm for pseudo-contractive mapping in Hilbert spaces as follows.

Let $C$ be a nonempty closed convex subset of a real Hilbert space $H$. Let $T: C \rightarrow C$ be a pseudocontraction. Let $\left\{\alpha_{n}\right\}$ be a sequence in $(0,1)$. Let $x_{0} \in H$. For $C_{1}=C$ and $x_{1}=P_{C_{1}}\left(x_{0}\right)$, define a sequence $\left\{x_{n}\right\}$ of $C$ as follows:

$$
\begin{gathered}
y_{n}=\left(1-\alpha_{n}\right) x_{n}+\alpha_{n} T x_{n}, \\
C_{n+1}=\left\{v \in C_{n}:\left\|\alpha_{n}(I-T) y_{n}\right\|^{2}\right. \\
\left.\leqslant 2 \alpha_{n}\left\langle x_{n}-v,(I-T) y_{n}\right\rangle\right\}, \\
x_{n+1}=P_{C_{n+1}}\left(x_{0}\right) .
\end{gathered}
$$

Theorem 3 (Yao et al. [29]). Let $C$ be a nonempty closed convex subset of a real Hilbert space $H$. Let $T: C \rightarrow C$ be an L-Lipschitz pseudocontraction such that $F(T) \neq \emptyset$. Assume that the sequence $\left\{\alpha_{n}\right\} \subset[a, b]$ for some $a, b \in(0,1 /(L+1))$. Then, the sequence $\left\{x_{n}\right\}$ generated by (9) converges strongly to $P_{F(T)}\left(x_{0}\right)$.

In 2009, Aoyama et al. [25] provided the useful and interesting lemma to confirm that the sequence generated by the shrinking projection method is well defined even if the firmly nonexpansive mapping $T$ has no fixed points.

Lemma 4 (Aoyama et al. [25, Lemma 4.2]). Let $H$ be a Hilbert space, $C$ a nonempty closed convex subset of $H, T: C \rightarrow C$ a firmly nonexpansive mapping, and $x_{0} \in H$. Let $\left\{x_{n}\right\}$ be a sequence in $C$ and $\left\{C_{n}\right\}$ a sequence of closed convex subsets of $H$ generated by $C_{1}=C$ and

$$
\begin{gathered}
x_{n}=P_{C_{n}}\left(x_{0}\right), \\
C_{n+1}=\left\{z \in C_{n}:\left\langle T x_{n}-z, x_{n}-T x_{n}\right\rangle \geq 0\right\}
\end{gathered}
$$

for all $n \in \mathbb{N}$. Then, $C_{n}$ is nonempty for every $n \in \mathbb{N}$, and, consequently, $\left\{x_{n}\right\}$ is well defined.

By using the lemma mentioned above, they proved the following theorem.

Theorem 5 (Aoyama et al. [25, Theorem 4.3]). Let $H$ be a Hilbert space, $C$ a nonempty closed convex subset of $H, T$ : $C \rightarrow C$ a firmly nonexpansive mapping and $x_{0} \in H$. Let $\left\{x_{n}\right\}$ be a sequence in $C$ and $\left\{C_{n}\right\}$ a sequence of closed convex subsets of $H$ generated by $C_{1}=C$ and

$$
\begin{gathered}
x_{n}=P_{C_{n}}\left(x_{0}\right), \\
C_{n+1}=\left\{z \in C_{n}:\left\langle T x_{n}-z, x_{n}-T x_{n}\right\rangle \geq 0\right\}
\end{gathered}
$$


for all $n \in \mathbb{N}$. Then, the following are equivalent:

(i) $\bigcap_{n=1}^{\infty} C_{n}$ is nonempty;

(ii) $\left\{x_{n}\right\}$ is bounded;

(iii) $F(T)$ is nonempty.

Motivated and inspired by the results mentioned above, in this paper, we provide some existence theorems of a strict pseudocontraction by the way of the shrinking projection method, involving some necessary and sufficient conditions. Then, we prove a strong convergence theorem and present its applications to confirm the existence of the zeros of an inverse strongly monotone operator along with its convergent results. tions:

Throughout the paper, we will using the following nota-

(i) $\rightarrow$ for strong convergence and $\rightarrow$ for weak convergence;

(ii) $\omega_{w}\left(x_{n}\right)=\left\{x: \exists x_{n_{i}} \rightarrow x\right\}$ denotes the weak $\omega$-limit set of $\left\{x_{n}\right\}$.

\section{Preliminaries}

In this section, some definitions are provided, and some relevant lemmas which are useful to prove in the next section are collected. Most of them are known and others are not hard to prove.

Let $H$ be a real Hilbert space with inner product $\langle\cdot, \cdot\rangle$ and norm $\|\cdot\|$, and let $C$ be a closed convex subset of $H$. For every point $x \in H$, there exists a unique nearest point in $C$, denoted by $P_{C}(x)$, such that

$$
\left\|x-P_{C} x\right\| \leqslant\|x-y\|, \quad \forall y \in C .
$$

The mapping $P_{C}$ is called the metric projection of $H$ onto $C$. It is well known that $P_{C}$ is a firmly nonexpansive mapping of $H$ onto $C$, that is,

$$
\left\|P_{C} x-P_{C} y\right\|^{2} \leq\left\langle P_{C} x-P_{C} y, x-y\right\rangle, \quad \forall x, y \in H .
$$

Furthermore, for any $x \in H$ and $z \in C$,

$$
z=P_{C} x \Longleftrightarrow\langle x-z, z-y\rangle \geq 0, \quad \forall y \in C .
$$

Moreover, $P_{C} x$ is characterized by the following:

$$
\|x-y\|^{2} \geq\left\|x-P_{C} x\right\|^{2}+\left\|y-P_{C} x\right\|^{2}, \quad \forall y \in C .
$$

It is obvious that the following equality holds for all $x, y \in H$ :

$$
\|x-y\|^{2}=\|x\|^{2}-\|y\|^{2}-2\langle x-y, y\rangle, \quad \forall x, y \in H .
$$

Proposition 6 (see [11, Proposition 2.1]). Assuming that $C$ is a closed convex subset of a Hilbert space $H$, let $T: C \rightarrow C$ be a self-mapping of $C$.

(i) If $T$ is a $k$-strict pseudocontraction, then $T$ satisfies the Lipschitz condition

$$
\|T x-T y\| \leqslant \frac{1+k}{1-k}\|x-y\| \quad \forall x, y \in C .
$$

(ii) If $T$ is a $k$-strictpseudocontraction, then $I-T$ is demiclosed at zero; that is, if $\left\{x_{n}\right\}$ is a sequence in $C$ such that $x_{n} \rightarrow z$ and $(I-T) x_{n} \rightarrow 0$, then $(I-T) z=0$.

(iii) If $T$ is a $k$-quasi-strict strict pseudocontraction, then the set of fixed points $F(T)$ is a closed convex subset of $C$.

Lemma 7. Assuming that $C$ is a closed convex subset of a Hilbert space $H$, let $T: C \rightarrow C$ be a self-mapping of $C$. Then, the following are equivalent:

(i) $T$ is a $k$-strict pseudocontraction;

(ii) $I-T$ is $(1-k) / 2$-inverse strongly monotone.

Lemma 8 (see [30, Theorem 7.1.8]). Let B be a bounded closed convex subset of a Hilbert space $H$ and $A: B \rightarrow H a$ continuous monotone mapping. Then, there exists an element $u_{0} \in B$ such that $\left\langle v-u_{0}, A u_{0}\right\rangle \geq 0$ for all $v \in K$.

\section{Main Result}

In this section, motivated by Aoyama et al. [25] (see also, Matsushita and Takahashi [31]), we discuss the existence of fixed point of a strict pseudocontraction by using the shrinking projection technique acting as the tool to guarantee the existence of fixed point of a strict pseudocontraction.

Every iteration process generated by the shrinking projection method for a $k$-strict pseudocontraction $T$ is well defined even if $T$ is a fixed point free.

Lemma 9. Let $H$ be a Hilbert space, $C$ a nonempty closed convex subset of $H, T: C \rightarrow C$ a $k$-strict pseudocontraction and $x_{0} \in H$. Let $\left\{x_{n}\right\}$ be a sequence in $C$ and $\left\{C_{n}\right\}$ a sequence of closed convex subsets of $H$ generated by $C_{1}=C$ and

$$
\begin{gathered}
x_{1}=P_{C_{1}}\left(x_{0}\right), \\
y_{n}=\left(1-\alpha_{n}\right) x_{n}+\alpha_{n} T x_{n}, \quad 0<\alpha_{n}<\frac{1-k}{2}, \\
C_{n+1}=\left\{z \in C_{n}:\left\|\alpha_{n}(I-T) y_{n}\right\|^{2}\right. \\
\left.\leq 2 \alpha_{n}\left[1-\frac{\alpha_{n}}{1-k}\right]\left\langle x_{n}-z,(I-T) y_{n}\right\rangle\right\}, \\
x_{n+1}=P_{C_{n+1}}\left(x_{0}\right),
\end{gathered}
$$

for all $n \in \mathbb{N}$. Then, $C_{n}$ is nonempty for every $n \in \mathbb{N}$, and, consequently, $\left\{x_{n}\right\}$ is well defined.

Proof. Clearly, $C_{1}$ is nonempty. Suppose that $C_{n}$ is nonempty for some $n \in \mathbb{N}$. Since $C_{n} \subset C_{n-1} \subset \cdots \subset C_{1}$, we have that $C_{1}, C_{2}, \ldots, C_{n}$ are nonempty and hence $\left\{x_{1}, x_{2}, \ldots, x_{n}\right\}$ is well defined. Put $r=\max \left\{\left\|y_{i}\right\|: i=1,2, \ldots, n\right\}$ and $B_{r}=\{z \in H:\|z\| \leq r\}$. Obviously, $C \cap B_{r}$ is a nonempty bounded closed convex subset of $H$. Let $I$ denote the identity mapping on $C$. Since $I-T$ is continuous and monotone, it follows from Lemma 8 that there exists $u \in C \cap B_{r}$ such that

$$
\langle y-u,(I-T) u\rangle \geq 0 \quad \forall y \in C \cap B_{r} .
$$


In particular, we have

$$
\left\langle y_{i}-u,(I-T) u\right\rangle \geq 0,
$$

for every $i=1,2 \ldots, n$. On the other hand, by employing the identity (16) and then adding and subtracting the terms $y_{i}$ and $(I-T) u$, we obtain

$$
\begin{aligned}
\| x_{i}- & u-\alpha_{i}(I-T) y_{i} \|^{2} \\
= & \left\|x_{i}-u\right\|^{2}-\left\|\alpha_{i}(I-T) y_{i}\right\|^{2} \\
& -2 \alpha_{i}\left\langle x_{i}-u-\alpha_{i}(I-T) y_{i},(I-T) y_{i}\right\rangle \\
= & \left\|x_{i}-u\right\|^{2}-\left\|\alpha_{i}(I-T) y_{i}\right\|^{2} \\
& -2 \alpha_{i}\left\langle x_{i}-y_{i}-\alpha_{i}(I-T) y_{i},(I-T) y_{i}\right\rangle \\
& -2 \alpha_{i}\left\langle y_{i}-u,(I-T) y_{i}\right\rangle \\
= & \left\|x_{i}-u\right\|^{2} \\
& -\left(\left\|\alpha_{i}(I-T) y_{i}\right\|^{2}\right. \\
& +2\left\langle x_{i}-y_{i}-\alpha_{i}(I-T) y_{i},\right. \\
& \left.\left.\alpha_{i}(I-T) y_{i}\right\rangle\right) \\
& -2 \alpha_{i}\left\langle y_{i}-u,(I-T) y_{i}-(I-T) u\right\rangle \\
- & 2 \alpha_{i}\left\langle y_{i}-u,(I-T) u\right\rangle .
\end{aligned}
$$

By using the identity (16) again, it follows that

$$
\begin{aligned}
\left\|\alpha_{i}(I-T) y_{i}\right\|^{2} \\
\quad+2\left\langle x_{i}-y_{i}-\alpha_{i}(I-T) y_{i}, \alpha_{i}(I-T) y_{i}\right\rangle \\
=\left\|\alpha_{i}(I-T) y_{i}\right\|^{2}+\left\|x_{i}-y_{i}\right\|^{2} \\
\quad-\left\|\alpha_{i}(I-T) y_{i}\right\|^{2} \\
\quad-\left\|x_{i}-y_{i}-\alpha_{i}(I-T) y_{i}\right\|^{2} \\
=\left\|x_{i}-y_{i}\right\|^{2}-\left\|x_{i}-y_{i}-\alpha_{i}(I-T) y_{i}\right\|^{2} .
\end{aligned}
$$

Substituting (22) in (21), we have

$$
\begin{aligned}
\| x_{i}- & u-\alpha_{i}(I-T) y_{i} \|^{2} \\
= & \left\|x_{i}-u\right\|^{2}-\left\|x_{i}-y_{i}\right\|^{2} \\
& +\left\|x_{i}-y_{i}-\alpha_{i}(I-T) y_{i}\right\|^{2} \\
& -2 \alpha_{i}\left\langle y_{i}-u,(I-T) y_{i}-(I-T) u\right\rangle \\
& -2 \alpha_{i}\left\langle y_{i}-u,(I-T) u\right\rangle \\
= & \left\|x_{i}-u\right\|^{2}-\alpha_{i}^{2}\left\|(I-T) x_{i}\right\|^{2} \\
& +\alpha_{i}^{2}\left\|(I-T) x_{i}-(I-T) y_{i}\right\|^{2}
\end{aligned}
$$

$$
\begin{aligned}
& -2 \alpha_{i}\left\langle y_{i}-u,(I-T) y_{i}-(I-T) u\right\rangle \\
& -2 \alpha_{i}\left\langle y_{i}-u,(I-T) u\right\rangle .
\end{aligned}
$$

By the virtue of Lemma 7 and some simple calculations, we obtain

$$
\begin{aligned}
& \left\|(I-T) x_{i}-(I-T) y_{i}\right\|^{2} \\
& \leq \frac{2}{1-k}\left\langle x_{i}-y_{i},(I-T) x_{i}-(I-T) y_{i}\right\rangle \\
& =\frac{2}{1-k}\left(\left\langle x_{i}-y_{i},(I-T) x_{i}\right\rangle\right. \\
& \left.-\left\langle x_{i}-y_{i},(I-T) y_{i}\right\rangle\right) \\
& =\frac{2}{1-k}\left(\alpha_{i}\left\|(I-T) x_{i}\right\|^{2}\right. \\
& -\left\langle x_{i}-u,(I-T) y_{i}\right\rangle \\
& \left.-\left\langle u-y_{i},(I-T) y_{i}\right\rangle\right) \\
& =\frac{2}{1-k}\left(\alpha_{i}\left\|(I-T) x_{i}\right\|^{2}\right. \\
& -\left\langle x_{i}-u,(I-T) y_{i}\right\rangle \\
& +\left\langle u-y_{i},\left((I-T) u-(I-T) y_{i}\right)\right. \\
& -(I-T) u\rangle) \\
& =\frac{2}{1-k}\left(\alpha_{i}\left\|(I-T) x_{i}\right\|^{2}\right. \\
& -\left\langle x_{i}-u,(I-T) y_{i}\right\rangle \\
& +\left\langle u-y_{i},(I-T) u-(I-T) y_{i}\right\rangle \\
& \left.+\left\langle y_{i}-u,(I-T) u\right\rangle\right) \text {. }
\end{aligned}
$$

Joining (23) for the term $\left\|(I-T) x_{i}-(I-T) y_{i}\right\|^{2}$ with (24) and by $0<\alpha_{n}<(1-k) / 2$, the monotonicity of $(I-T)$, and (20), we have

$$
\begin{aligned}
& \left\|x_{i}-u-\alpha_{i}(I-T) y_{i}\right\|^{2} \\
& \leq\left\|x_{i}-u\right\|^{2}-\alpha_{i}^{2}\left\|(I-T) x_{i}\right\|^{2} \\
& +\alpha_{i}^{2} \frac{2}{1-k}\left(\alpha_{i}\left\|(I-T) x_{i}\right\|^{2}\right. \\
& -\left\langle x_{i}-u,(I-T) y_{i}\right\rangle \\
& +\left\langle u-y_{i},(I-T) u\right. \\
& \left.\quad-(I-T) y_{i}\right\rangle \\
& \left.+\left\langle y_{i}-u,(I-T) u\right\rangle\right)
\end{aligned}
$$




$$
\begin{aligned}
& -2 \alpha_{i}\left\langle y_{i}-u,(I-T) y_{i}-(I-T) u\right\rangle \\
& -2 \alpha_{i}\left\langle y_{i}-u,(I-T) u\right\rangle \\
= & \left\|x_{i}-u\right\|^{2} \\
& +\alpha_{i}^{2}\left(\frac{2 \alpha_{i}}{1-k}-1\right)\left\|(I-T) x_{i}\right\|^{2} \\
& -\frac{2 \alpha_{i}^{2}}{1-k}\left\langle x_{i}-u,(I-T) y_{i}\right\rangle \\
& +2 \alpha_{i}\left(\frac{\alpha_{i}}{1-k}-1\right) \\
& \times\left\langle u-y_{i},(I-T) u-(I-T) y_{i}\right\rangle \\
& +2 \alpha_{i}\left(\frac{\alpha_{i}}{1-k}-1\right)\left\langle y_{i}-u,(I-T) u\right\rangle \\
\leq & \left\|x_{i}-u\right\|^{2}-\frac{2 \alpha_{i}^{2}}{1-k}\left\langle x_{i}-u,(I-T) y_{i}\right\rangle .
\end{aligned}
$$

Notice that

$$
\begin{aligned}
\left\|x_{i}-u-\alpha_{i}(I-T) y_{i}\right\|^{2} \\
=\left\|x_{i}-u\right\|^{2}-2\left\langle x_{i}-u, \alpha_{i}(I-T) y_{i}\right\rangle \\
\quad+\left\|\alpha_{i}(I-T) y_{i}\right\|^{2} .
\end{aligned}
$$

Combining (25) and (26), we have

$$
\left\|\alpha_{i}(I-T) y_{i}\right\|^{2} \leq 2 \alpha_{i}\left[1-\frac{\alpha_{i}}{1-k}\right]\left\langle x_{i}-u,(I-T) y_{i}\right\rangle
$$

for every $i=1,2, \ldots, n$. This shows that $u \in C_{n+1}$. By induction on $n$, we obtain the desired result.

The following theorem provides some necessary and sufficient conditions to confirm the existence of a fixed point of a strict pseudocontraction in Hilbert spaces.

Theorem 10. Let all the assumptions be as in Lemma 9 and $0<a \leq \alpha_{n} \leq b<(1-k) / 2$ for all $n \in \mathbb{N}$. Then, the following are equivalent:

(i) $\bigcap_{n=1}^{\infty} C_{n}$ is nonempty;

(ii) $\left\{x_{n}\right\}$ is bounded;

(iii) $F(T)$ is nonempty.

Proof. [(i) $\Rightarrow$ (ii)] By letting $u \in \bigcap_{n=1}^{\infty} C_{n}$, it follows from the nonexpansiveness of $P_{C_{n}}$ that

$$
\left\|x_{n}-u\right\| \leq\left\|P_{C_{n}} x_{0}-P_{C_{n}} u\right\| \leq\left\|x_{0}-u\right\| .
$$

This shows that $\left\{x_{n}\right\}$ is bounded. [(ii) $\Rightarrow$ (iii)] Suppose that $\left\{x_{n}\right\}$ is bounded; we observe that

$$
\begin{aligned}
0 & \leq\left\|x_{n+1}-x_{n}\right\|^{2} \\
& =\left\|x_{n+1}-P_{C_{n}} x_{0}\right\|^{2} \\
& \leq\left\|x_{n+1}-x_{0}\right\|^{2}-\left\|P_{C_{n}} x_{0}-x_{0}\right\|^{2} \\
& =\left\|x_{n+1}-x_{0}\right\|^{2}-\left\|x_{n}-x_{0}\right\|^{2} .
\end{aligned}
$$

This shows that $\left\{\left\|x_{n}-x_{0}\right\|\right\}$ is nondecreasing and then with the boundedness of $\left\{x_{n}\right\}$, we have that $\lim _{n \rightarrow \infty}\left\|x_{n}-x_{0}\right\|$ exists. By using (29), we obtain

$$
\left\|x_{n+1}-x_{n}\right\| \longrightarrow 0 \text { as } n \longrightarrow \infty \text {. }
$$

Since $x_{n+1} \in C_{n+1}$ and $0<a \leq \alpha_{n} \leq b<(1-k) / 2$, we have

$$
\begin{aligned}
& \left\|\alpha_{n}(I-T) y_{n}\right\|^{2} \\
& \qquad 2 \alpha_{n}\left[1-\frac{\alpha_{n}}{1-k}\right] \\
& \quad \times\left\langle x_{n}-x_{n+1},(I-T) y_{n}\right\rangle \longrightarrow 0 \text { as } n \longrightarrow \infty .
\end{aligned}
$$

Since $\left\{\alpha_{n}\right\} \subset[a, b]$, we obtain

$$
\left\|y_{n}-T y_{n}\right\| \longrightarrow 0 \quad \text { as } n \longrightarrow \infty .
$$

Furthermore, Proposition 6 (i) allows us to have

$$
\begin{aligned}
&\left\|x_{n}-T x_{n}\right\| \\
& \leq\left\|x_{n}-y_{n}\right\|+\left\|y_{n}-T y_{n}\right\| \\
&+\left\|T y_{n}-T x_{n}\right\| \\
& \leq\left\|x_{n}-y_{n}\right\|+\left\|y_{n}-T y_{n}\right\| \\
&+\left(\frac{1+k}{1-k}\right)\left\|y_{n}-x_{n}\right\| \\
&= \frac{2 \alpha_{n}}{1-k}\left\|x_{n}-T x_{n}\right\|+\left\|y_{n}-T y_{n}\right\| .
\end{aligned}
$$

By simple calculation, we have

$$
\begin{aligned}
&\left\|x_{n}-T x_{n}\right\| \leq\left(\frac{1}{1-2 \alpha_{n} /(1-k)}\right)\left\|y_{n}-T y_{n}\right\| \\
& \leq\left(\frac{1}{1-2 b /(1-k)}\right)\left\|y_{n}-T y_{n}\right\| \longrightarrow 0 \\
& \text { as } n \longrightarrow \infty .
\end{aligned}
$$

Since $\left\{x_{n}\right\}$ is bounded, the reflexivity of $H$ allows a subsequence $\left\{x_{n_{i}}\right\}$ of $\left\{x_{n}\right\}$ such that $x_{n_{i}} \rightarrow p \in C$ as $i \rightarrow \infty$. By using (34) and the demicloseness of $(I-T)$, we obtain $p-T p=$ 0 ; that is, $p \in F(T) \neq \emptyset$.

[(iii) $\Rightarrow($ i) ] Suppose that $F(T) \neq \emptyset$. We will show that $F(T) \subset C_{n}$ for every $n \in \mathbb{N}$. Let $p \in F(T)$; then we have $(I-T) p=0$. Let us replace $u$ in the proof of Lemma 9 with $p$; it is not difficult to see that all equalities and inequalities are satisfied until (27). This implies that $p \in C_{n}$ for all $n \in \mathbb{N}$. Therefore, $F(T) \subset \cap_{n=1}^{\infty} C_{n} \neq \emptyset$. 
Theorem 11. Let all the assumptions be as in Theorem 10. If $\bigcap_{n=1}^{\infty} C_{n} \neq \emptyset\left(\Leftrightarrow\left\{x_{n}\right\}\right.$ is bounded $\left.\Leftrightarrow F(T) \neq \emptyset\right)$, then the sequence $\left\{x_{n}\right\}$ generated by (18) converges strongly to some points of $C$, and its strong limit point is a member of $F(T)$; that is, $\lim _{n \rightarrow \infty} x_{n}=P_{F(T)} x_{0} \in F(T)$.

Proof. If $\bigcap_{n=1}^{\infty} C_{n} \neq \emptyset$, then Theorem 10 guarantees that $\left\{x_{n}\right\}$ is bounded and $\lim _{n \rightarrow \infty}\left\|x_{n}-x_{0}\right\|$ exists. So, there is $\left\{x_{n_{n}}\right\} \subset\left\{x_{n}\right\}$ such that $x_{n_{i}} \rightarrow p \in C$ as $i \rightarrow \infty$. By using (34) and the demicloseness of $(I-T)$, we obtain $p \in F(T)$. On the other hand, noticing that $P_{F(T)} x_{0} \in F(T) \subset C_{n}$, we observe that

$$
\left\|x_{n}-x_{0}\right\|=\left\|P_{C_{n}} x_{0}-x_{0}\right\| \leq\left\|P_{F(T)} x_{0}-x_{0}\right\|
$$

for every $n \in \mathbb{N}$. Since $\|\cdot\|^{2}$ is weakly lower semicontinuous and $\left\{\left\|x_{n}-x_{0}\right\|\right\}$ is convergent, it follows from (35) that

$$
\begin{aligned}
\left\|p-x_{0}\right\|^{2} & \leq \liminf _{i \rightarrow \infty}\left\|x_{n_{i}}-x_{0}\right\|^{2} \\
& =\lim _{n \rightarrow \infty}\left\|x_{n}-x_{0}\right\|^{2} \leq\left\|P_{F(T)} x_{0}-x_{0}\right\|^{2} .
\end{aligned}
$$

Taking into account $p \in F(T)$, we obtain $p=P_{F(T)} x_{0}$. This implies that $x_{n} \rightarrow P_{F(T)} x_{0}$ and $\left\|x_{n}-x_{0}\right\| \rightarrow\left\|P_{F(T)} x_{0}-x_{0}\right\|$. Hence, by using (16), we obtain

$$
\begin{aligned}
\| x_{n} & -P_{F(T)} x_{0} \|^{2} \\
= & \left\|x_{n}-x_{0}-\left(P_{F(T)} x_{0}-x_{0}\right)\right\|^{2} \\
= & \left\|x_{n}-x_{0}\right\|^{2}-\left\|P_{F(T)} x_{0}-x_{0}\right\|^{2} \\
& \quad-2\left\langle x_{n}-P_{F(T)} x_{0}, P_{F(T)} x_{0}-x_{0}\right\rangle \longrightarrow 0 .
\end{aligned}
$$

This completes the proof.

\section{Deduced Theorems and Applications}

In this section, some deduced theorems and applications of the main theorem are provided in order to guarantee the existence of fixed points of a nonexpansive mapping and the existence of the zeros of an inverse strongly monotone operator. Moreover, we also have the methods that can be used to find fixed points and zero points as mentioned above.

If $T: C \rightarrow C$ is nonexpansive $(\Leftrightarrow T$ is a 0 -strict pseudocontraction), then we have the following corollaries.

Corollary 12. Let $H$ be a Hilbert space, $C$ a nonempty closed convex subset of $H, T: C \rightarrow C$ a nonexpansive mapping, and $x_{0} \in H$. Let $\left\{x_{n}\right\}$ be a sequence in $C$ and $\left\{C_{n}\right\}$ a sequence of closed convex subsets of $H$ generated by $C_{1}=C$ and

$$
\begin{gathered}
x_{1}=P_{C_{1}} x_{0}, \\
y_{n}=\left(1-\alpha_{n}\right) x_{n}+\alpha_{n} T x_{n}, \\
0<a \leq \alpha_{n} \leq b<\frac{1}{2},
\end{gathered}
$$

$$
\begin{gathered}
C_{n+1}=\left\{z \in C_{n}:\left\|\alpha_{n}(I-T) y_{n}\right\|^{2}\right. \\
\left.\leq 2 \alpha_{n}\left(1-\alpha_{n}\right)\left\langle x_{n}-z,(I-T) y_{n}\right\rangle\right\}, \\
x_{n+1}=P_{C_{n+1}} x_{0}
\end{gathered}
$$

for all $n \in \mathbb{N}$. Then, the following are equivalent:

(i) $\bigcap_{n=1}^{\infty} C_{n}$ is nonempty;

(ii) $\left\{x_{n}\right\}$ is bounded;

(iii) $F(T)$ is nonempty.

Corollary 13. Let $H$ be a Hilbert space, $C$ a nonempty closed convex subset of $H, T: C \rightarrow C$ a nonexpansive mapping, and $x_{0} \in H$. Let $\left\{x_{n}\right\}$ be a sequence in $C$ and $\left\{C_{n}\right\}$ a sequence of closed convex subsets of $H$ generated by $C_{1}=C$ and

$$
\begin{gathered}
x_{1}=P_{C_{1}} x_{0}, \\
y_{n}=\left(1-\alpha_{n}\right) x_{n}+\alpha_{n} T x_{n} ; \quad 0<a \leq \alpha_{n} \leq b<\frac{1}{2}, \\
C_{n+1}=\left\{z \in C_{n}:\left\|\alpha_{n}(I-T) y_{n}\right\|^{2}\right. \\
\left.\leq 2 \alpha_{n}\left(1-\alpha_{n}\right)\left\langle x_{n}-z,(I-T) y_{n}\right\rangle\right\}, \\
x_{n+1}=P_{C_{n+1}} x_{0}
\end{gathered}
$$

for all $n \in \mathbb{N}$. If $\bigcap_{n=1}^{\infty} C_{n} \neq \emptyset\left(\Leftrightarrow\left\{x_{n}\right\}\right.$ is bounded $)$, then $\left\{x_{n}\right\}$ converges strongly to some points of $C$, and its strong limit point is a member of $F(T)$; that is, $\lim _{n \rightarrow \infty} x_{n}=P_{F(T)}\left(x_{0}\right) \in F(T)$ (i.e., $F(T) \neq \emptyset$ ).

Recall that a mapping $A$ is said to be monotone if $\langle x-$ $y, A x-A y\rangle \geqslant 0$ for all $x, y \in H$ and inverse strongly monotone if there exists a real number $\gamma>0$ such that $\langle x-y, A x-A y\rangle \geqslant \gamma\|A x-A y\|^{2}$ for all $x, y \in H$. For the second case, $A$ is said to be $\gamma$-inverse strongly monotone. It follows immediately that if $A$ is $\gamma$-inverse strongly monotone, then $A$ is the Lipschitz continuous; that is, $\|A x-A y\| \leqslant$ $(1 / \gamma)\|x-y\|$. It is well known (see, e.g., [32]) that if $A$ is monotone, then the solutions of the equation $A x=0$ correspond to the equilibrium points of some evolution systems. Therefore, it is important to focus on finding the zero point of monotone mappings. The pseudo-contractive mapping and strictly pseudo-contractive mapping are strongly related to the monotone mapping and the inverse strongly monotone mapping, respectively. It is well known that

(i) $A$ is monotone $\Leftrightarrow T:=(I-A)$ is pseudo-contractive.

(ii) $A$ is inverse strongly monotone $\Leftrightarrow T:=(I-A)$ is strictly pseudo-contractive. 
Indeed, for (ii), we notice that the following equality always holds in a real Hilbert space:

$$
\begin{aligned}
&\|(I-A) x-(I-A) y\|^{2} \\
&=\|x-y\|^{2}+\|A x-A y\|^{2} \\
& \quad-2\langle x-y, A x-A y\rangle \quad \forall x, y \in H .
\end{aligned}
$$

Without loss of generality, we can assume that $\gamma \in(0,1 / 2]$ and then it yields that

$$
\begin{aligned}
& \langle x-y, A x-A y\rangle \\
& \geqslant \gamma\|A x-A y\|^{2} \\
& \Longleftrightarrow-2\langle x-y, A x-A y\rangle \\
& \leqslant-2 \gamma\|A x-A y\|^{2} \\
& \Longleftrightarrow\|(I-A) x-(I-A) y\|^{2} \\
& \leqslant\|x-y\|^{2}+(1-2 \gamma) \\
& \quad \times\|A x-A y\|^{2} \quad(\operatorname{via}(40)) \\
& \Longleftrightarrow\|T x-T y\|^{2} \leqslant\|x-y\|^{2} \\
& \quad+\kappa\|(I-T) x-(I-T) y\|^{2}
\end{aligned}
$$

(where $T:=(I-A), \kappa:=1-2 \gamma)$.

Every iteration process generated by the shrinking projection method for a $\gamma$-inverse strongly monotone $A$ is well defined even if $A$ has no zeros.

Lemma 14. Let $H$ be a Hilbert space, and let $A: H \rightarrow H$ be $\gamma$-inverse strongly monotone (without loss of generality, one can assume that $\gamma \in(0,1 / 2])$. Let $x_{0} \in H, C_{1}=C$ and $\left\{x_{n}\right\} a$ sequence generated by

$$
\begin{gathered}
x_{1}=P_{C_{1}} x_{0}, \\
y_{n}=\left(I-\alpha_{n} A\right) x_{n}, \quad 0<\alpha_{n}<\gamma, \\
C_{n+1}=\left\{z \in C_{n}:\left\|\alpha_{n} A y_{n}\right\|^{2}\right. \\
\left.\leq 2 \alpha_{n}\left[1-\frac{\alpha_{n}}{2 \gamma}\right]\left\langle x_{n}-z, A y_{n}\right\rangle\right\}, \\
x_{n+1}=P_{C_{n+1}} x_{0}
\end{gathered}
$$

for all $n \in \mathbb{N}$. Then, $C_{n}$ is nonempty for every $n \in \mathbb{N}$, and, consequently, $\left\{x_{n}\right\}$ is well defined.

Proof. Let $T:=(I-A)$. Then, $T$ is $(1-2 \gamma)$-pseudocontraction,

$$
\begin{aligned}
y_{n} & =\left(I-\alpha_{n} A\right) x_{n} \\
& =\left(I-\alpha_{n}(I-(I-A))\right) x_{n} \\
& =\left(1-\alpha_{n}\right) x_{n}+\alpha_{n} T x_{n},
\end{aligned}
$$

and $2 \gamma=1-(1-2 \gamma)=1-k$, where $k:=1-2 \gamma$. Hence, by applying Lemma 9, we have the desired result.

The following theorem provides some necessary and sufficient conditions to confirm the existence of the zeros of $\gamma$-inverse strongly monotone in Hilbert spaces.

Theorem 15. Let all the assumptions be as in Lemma 14 and $0<a \leq \alpha_{n} \leq b<\gamma$ for all $n \in \mathbb{N}$. Then, the following are equivalent:

(i) $\bigcap_{n=1}^{\infty} C_{n}$ is nonempty;

(ii) $\left\{x_{n}\right\}$ is bounded;

(iii) $A^{-1}(0)$ is nonempty.

Proof. Let $T:=(I-A)$. Then, $T$ is $(1-2 \gamma)$-pseudocontraction; it is not difficult to show that $F(T)=A^{-1}(0)$ and $\gamma=(1-k) / 2$, where $k:=1-2 \gamma$. Hence, by applying Theorem 10, we have the desired result.

Theorem 16. Let all the assumptions be as in Theorem 15 . If $\bigcap_{n=1}^{\infty} C_{n} \neq \emptyset\left(\Leftrightarrow\left\{x_{n}\right\}\right.$ is bounded $\left.\Leftrightarrow A^{-1}(0) \neq \emptyset\right)$, then the sequence $\left\{x_{n}\right\}$ generated by (42) converges strongly to some points of $H$, and its strong limit point is a member of $A^{-1}(0)$; that is, $\lim _{n \rightarrow \infty} x_{n}=P_{A^{-1}(0)} x_{0} \in A^{-1}(0)$.

Proof. Let $T:=(I-A)$, and by applying Theorem 11, we have the desired result.

\section{Acknowledgments}

The author would like to thank Professor Dr. Naseer Shahzad and an anonymous referee for their valuable comments and suggestions, which were helpful in improving the paper. Moreover, the author would like to thank the Centre of Excellence in Mathematics under the Commission of Higher Education, Ministry of Education, Thailand. The project was supported by the Centre of Excellence in Mathematics, CHE, Si Ayutthaya Road, Bangkok 10400, Thailand.

\section{References}

[1] W. R. Mann, "Mean value methods in iteration," Proceedings of the American Mathematical Society, vol. 4, pp. 506-510, 1953.

[2] A. Genel and J. Lindenstrauss, "An example concerning fixed points," Israel Journal of Mathematics, vol. 22, no. 1, pp. 81-86, 1975.

[3] K. Nakajo and W. Takahashi, "Strong convergence theorems for nonexpansive mappings and nonexpansive semigroups," Journal of Mathematical Analysis and Applications, vol. 279, no. 2, pp. 372-379, 2003.

[4] K. Aoyama and W. Takahashi, "Strong convergence theorems for a family of relatively nonexpansive mappings in Banach spaces," Fixed Point Theory, vol. 8, no. 2, pp. 143-160, 2007.

[5] H. H. Bauschke and P. L. Combettes, "A weak-to-strong convergence principle for Fejér-monotone methods in Hilbert spaces," Mathematics of Operations Research, vol. 26, no. 2, pp. 248-264, 2001. 
[6] C. Jaiboon and P. Kumam, "Strong convergence theorems for solving equilibrium problems and fixed point problems of $\varepsilon$ strict pseudo-contraction mappings by two hybrid projection methods," Journal of Computational and Applied Mathematics, vol. 234 , no. 3, pp. 722-732, 2010.

[7] A. Jarernsuk and K. Ungchittrakool, "Strong convergence by a hybrid algorithm for solving equilibrium problem and fixed point problem of a Lipschitz pseudo-contraction in Hilbert spaces," Thai Journal of Mathematics, vol. 10, no. 1, pp. 181-194, 2012.

[8] S. Kamimura and W. Takahashi, "Strong convergence of a proximal-type algorithm in a Banach space," SIAM Journal on Optimization, vol. 13, no. 3, pp. 938-945, 2002.

[9] F. Kohsaka and W. Takahashi, "Strong convergence of an iterative sequence for maximal monotone operators in a Banach space," Abstract and Applied Analysis, no. 3, pp. 239-249, 2004.

[10] F. Kohsaka and W. Takahashi, "Approximating common fixed points of countable families of strongly nonexpansive mappings," Nonlinear Studies, vol. 14, no. 2, pp. 219-234, 2007.

[11] G. Marino and H. K. Xu, "Weak and strong convergence theorems for strict pseudo-contractions in Hilbert spaces," Journal of Mathematical Analysis and Applications, vol. 329, no. 1, pp. 336-346, 2007.

[12] C. Martinez-Yanes and H. K. Xu, "Strong convergence of the CQ method for fixed point iteration processes," Nonlinear Analysis A, vol. 64, no. 11, pp. 2400-2411, 2006.

[13] S. Y. Matsushita and W. Takahashi, "A strong convergence theorem for relatively nonexpansive mappings in a Banach space," Journal of Approximation Theory, vol. 134, no. 2, pp. 257266, 2005.

[14] S. Plubtieng and T. Thammathiwat, "Shrinking projection methods for a family of relatively nonexpansive mappings, equilibrium problems and variational inequality problems in Banach spaces," Journal of Nonlinear Analysis and Optimization, vol. 1, no. 1, pp. 97-110, 2010.

[15] S. Ohsawa and W. Takahashi, "Strong convergence theorems for resolvents of maximal monotone operators in Banach spaces," Archiv der Mathematik, vol. 81, no. 4, pp. 439-445, 2003.

[16] S. Saewan and P. Kumam, "The shrinking projection method for solving generalized equilibrium problems and common fixed points for asymptotically quasi- $\phi$-nonexpansive mappings," Fixed Point Theory and Applications, vol. 2011, article 9, 2011.

[17] M. V. Solodov and B. F. Svaiter, "Forcing strong convergence of proximal point iterations in a Hilbert space," Mathematical Programming A, vol. 87, no. 1, pp. 189-202, 2000.

[18] W. Takahashi, Y. Takeuchi, and R. Kubota, "Strong convergence theorems by hybrid methods for families of nonexpansive mappings in Hilbert spaces," Journal of Mathematical Analysis and Applications, vol. 341, no. 1, pp. 276-286, 2008.

[19] K. Ungchittrakool, "A strong convergence theorem for a common fixed points of two sequences of strictly pseudocontractive mappings in Hilbert spaces and applications," Abstract and Applied Analysis, vol. 2010, Article ID 876819, 17 pages, 2010.

[20] K. Ungchittrakool, "Strong convergence by a hybrid algorithm for finding a common fixed point of Lipschitz pseudocontraction and strict pseudocontraction in Hilbert spaces," Abstract and Applied Analysis, vol. 2011, Article ID 530683, 14 pages, 2011.

[21] K. Ungchittrakool, "An iterative shrinking projection method for solving fixed point problems of closed and $\phi$-strict pseudocontractions along with generalized mixed equilibrium problems in Banach spaces," Abstract and Applied Analysis, vol. 2012, Article ID 536283, 20 pages, 2012.
[22] K. Ungchittrakool and A. Jarernsuk, "Strong convergence by a hybrid algorithm for solving generalized mixed equilibrium problems and fixed point problems of a Lipschitz pseudocontraction in Hilbert spaces," Fixed Point Theory and Applications, vol. 2012, article 147, 14 pages, 2012.

[23] R. Wangkeeree and R. Wangkeeree, "The shrinking projection method for solving variational inequality problems and fixed point problems in Banach spaces," Abstract and Applied Analysis, vol. 2009, Article ID 624798, 26 pages, 2009.

[24] K. Aoyama, F. Kohsaka, and W. Takahashi, "Strong convergence theorems by shrinking and hybrid projection methods for relatively nonexpansive mappings in Banach spaces," in Nonlinear Analysis and Convex Analysis, pp. 7-26, Yokohama Publishers, Yokohama, Japan, 2009.

[25] K. Aoyama, F. Kohsaka, and W. Takahashi, "Shrinking projection methods for firmly nonexpansive mappings," Nonlinear Analysis A, vol. 71, no. 12, pp. e1626-e1632, 2009.

[26] O. Scherzer, "Convergence criteria of iterative methods based on Landweber iteration for solving nonlinear problems," Journal of Mathematical Analysis and Applications, vol. 194, no. 3, pp. 911-933, 1995.

[27] F. E. Browder and W. V. Petryshyn, "Construction of fixed points of nonlinear mappings in Hilbert space," Journal of Mathematical Analysis and Applications, vol. 20, pp. 197-228, 1967.

[28] H. Zhou, "Convergence theorems of fixed points for $k$-strict pseudo-contractions in Hilbert spaces," Nonlinear Analysis A, vol. 69, no. 2, pp. 456-462, 2008.

[29] Y. Yao, Y. C. Liou, and G. Marino, "A hybrid algorithm for pseudo-contractive mappings," Nonlinear Analysis A, vol. 71, no. 10, pp. 4997-5002, 2009.

[30] W. Takahashi, Nonlinear Functional Analysis, Yokohama Publishers, Yokohama, Japan, 2000.

[31] S. Y. Matsushita and W. Takahashi, "A proximal-type algorithm by the hybrid method for maximal monotone operators in a Banach space," in Nonlinear Analysis and Convex Analysis, pp. 355-365, Yokohama Publishers, Yokohama, Japan, 2007.

[32] K. Deimling, "Zeros of accretive operators," Manuscripta Mathematica, vol. 13, pp. 365-374, 1974. 


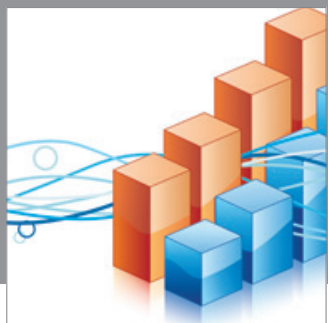

Advances in

Operations Research

mansans

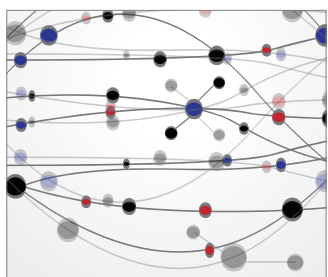

The Scientific World Journal
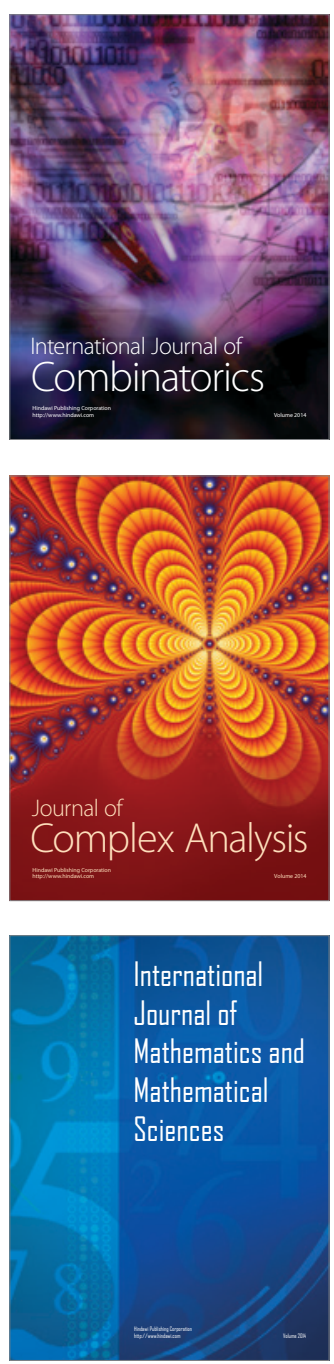
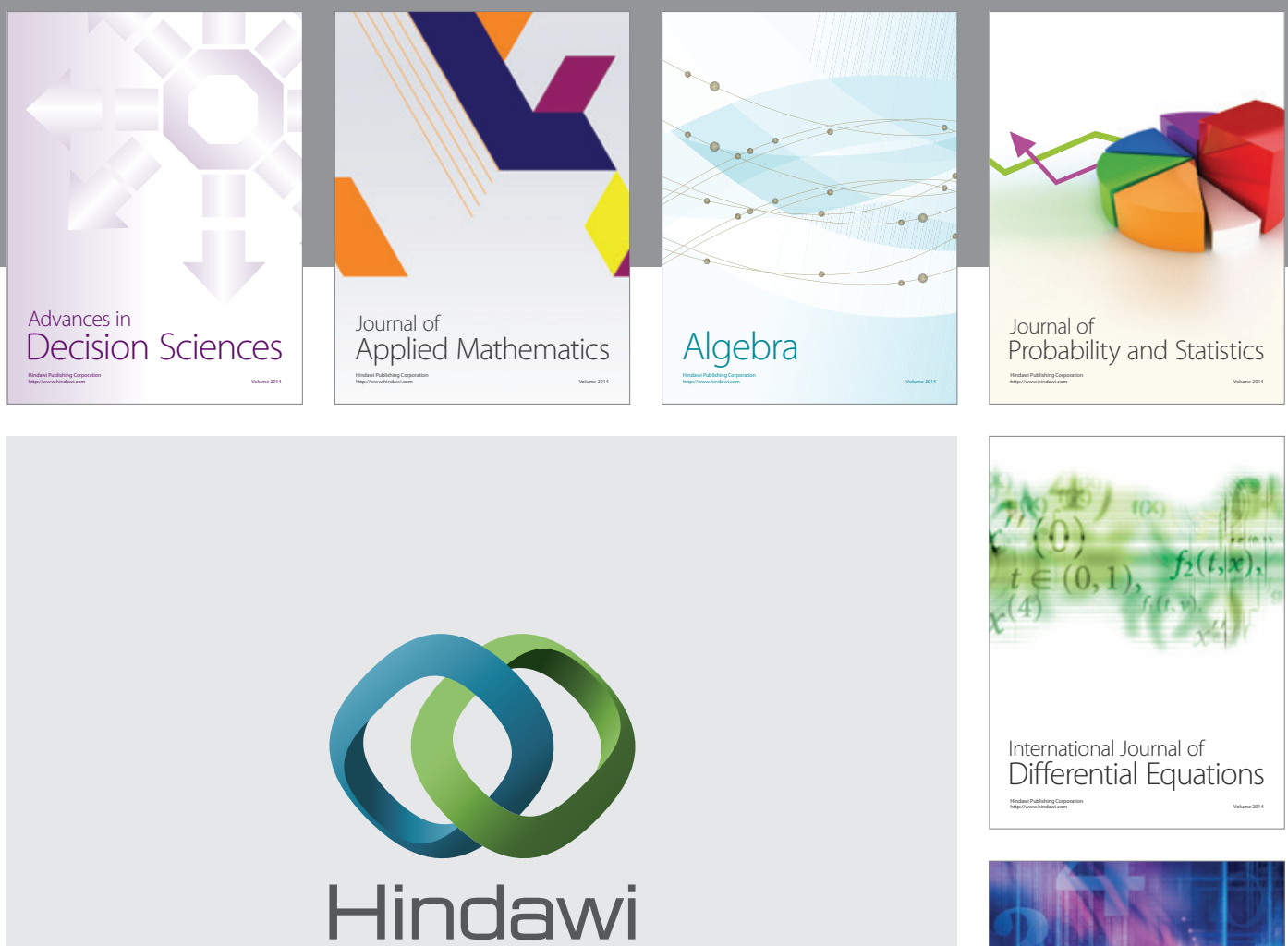

Submit your manuscripts at http://www.hindawi.com
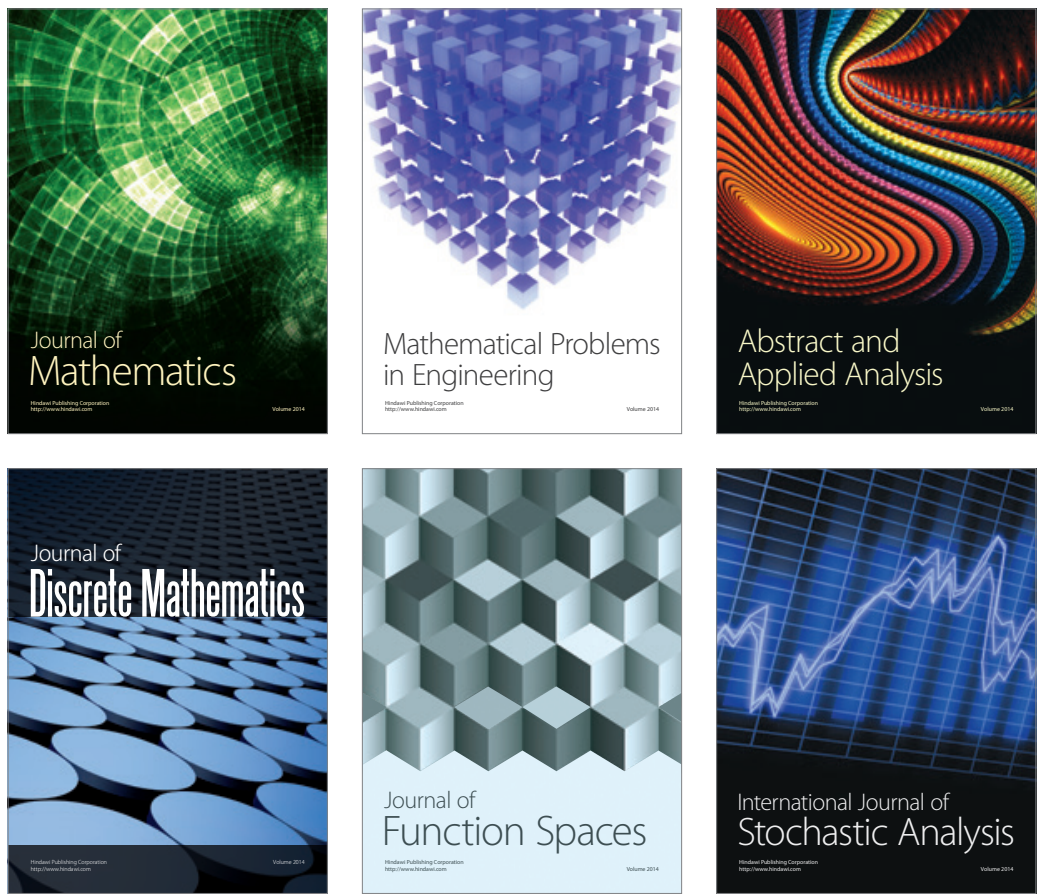

Journal of

Function Spaces

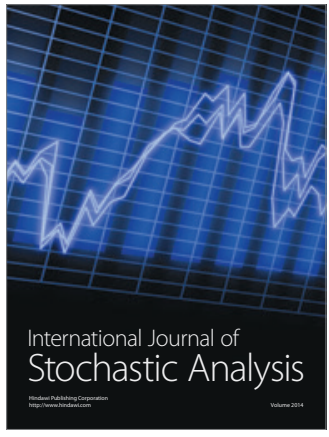

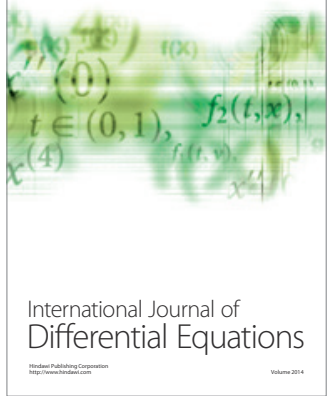
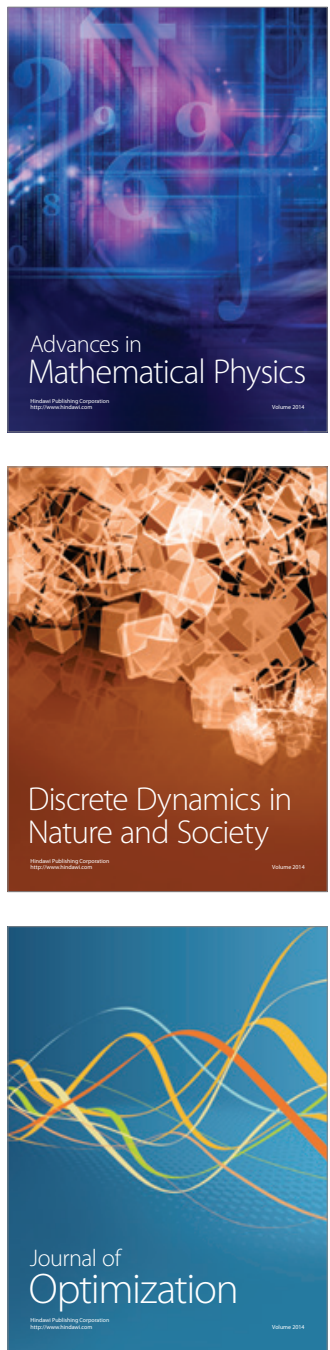